\title{
A função de produção da agropecuária gaúcha em 2006
}

\author{
Gustavo Inácio de Moraes* \\ Alexandre Rodrigues Loures**
}

\begin{abstract}
Resumo
O presente artigo estima uma função de produção para a agropecuária gaúcha a partir de dados do Censo 2006. Com base nessa função de produção estimada, obtêm-se as relações de complementariedade e de substituição entre os principais fatores produtivos. Destacadamente, relações de complementariedade inelásticas são obtidas entre área e trabalho; trabalho e tratores; e área e insumos de produção. A relação de substituição inelástica é encontrada para trabalho e outros insumos. Finalmente, nota-se relação de substituição elástica para área e tratores e tratores e outros insumos.
\end{abstract}

Palavras-chave: Agricultura. Função de produção. Rio Grande do Sul. Elasticidades de substituição.

* Professor do PPGE/PUCRS (Programa de Pós Graduação em Economia - Pontifícia Universidade Católica do Rio Grande do Sul). Email: gustavo.moraes@pucrs.br.

** Mestre em Economia pela Pontifícia Universidade Católica do Rio Grande do Sul e doutorando na Universidade Federal da Paraíba. Email: alexandre.loures@ymail.com.

http://dx.doi.org/10.5335/rtee.v0i41.3735

Submissão: 03/05/2013. Aceite: 05/07/2013 


\section{Introdução}

A agropecuária gaúcha passou por importantes transformações ao longo das décadas recentes. Sua expressão no valor adicionado bruto do Rio Grande do Sul não é tão significante quanto em outrora, mas ainda é uma importante fonte de riqueza regional, totalizando $9,86 \%$ da economia gaúcha em 2009 , ou $\mathrm{R} \$ 18,6$ bilhões ${ }^{1}$ (IBGE, 2011).

Desde os anos 1970, nota-se um decréscimo das áreas de pastagens, um aumento nas áreas de lavouras e, também, mais importante, nas áreas de matas e florestas. Ainda assim, a agropecuária gaúcha foi capaz de manter sua produtividade em permanente crescimento, ao menos se considerarmos a utilização de terras. A força de trabalho atualmente alocada para as atividades agropecuárias encontra-se em patamar inferior ao do início dos anos setenta, ainda que, durante os anos 1980, sua quantidade fosse crescente. Em paralelo, evidencia-se que a produtividade do trabalho também cresceu no período e contribuiu para que a economia agrícola do estado se fortalecesse. Outro indicador que demonstra a maior produtividade da agricultura regional é demonstrado pelo crescente uso de tratores, que atinge um total de 161,4 mil máquinas em 2006 (IBGE, 2007), tornando o estado um dos mais mecanizados, proporcionalmente à área, do país.

Ademais, a atividade agrícola local é uma importante absorvedora de mão de obra e, assim, cumpre também uma importante função socioeconômica ao garantir o rendimento e o sustento de inúmeras famílias. $\mathrm{O}$ censo agropecuário aponta que 1,219 milhão trabalhavam na agropecuária em 2006 , número equivalente a quase $11 \%$ da população, sendo superior aos $6,5 \%$ da força de trabalho concentrada na agricultura em território brasileiro (IBGE, 2008).

A Tabela 1 demonstra a evolução dos principais indicadores da agropecuária gaúcha ao longo dos diferentes censos agrícolas. Na Tabela 2, esses números são confrontados entre si, perfazendo indicadores propostos de produtividade. A evolução da produtividade da agricultura gaúcha é evidente desde os anos 1970. Contudo, algum efeito relativo ao difícil ano de 2005 pode ser observado nos dados relativos a 2006 , por exemplo, a queda representativa na quantidade de tratores por área disponibilizada para lavouras, conforme tabela 2. Entretanto, mesmo essa queda não foi capaz de interromper o contínuo decréscimo na relação de trabalhadores por trator.

Em especial, enquanto há trinta anos um trabalhador, em média, correspondia a 3 hectares de áreas de lavouras, hoje temos um indicador próximo a 6 hectares por trabalhador. No caso de tratores, o salto de produtividade foi mais duradouro e contínuo, ao menos até 1995. Em especial, o ritmo de incorporação de tratores à 
produção foi mais intenso do que a evolução no número de trabalhadores, sugerindo uma substituição, em sintonia com o processo econômico, destes por aqueles.

De qualquer maneira, essas são impressões relativas a uma evolução temporal. Com base nos dados do censo agropecuário realizado pelo IBGE em 2006, é possível examinar o problema da inter-relação entre os insumos de um ponto de vista geográfico. Por essa proposta, da perspectiva de amostragem dos dados de cada um dos municípios gaúchos, é possível estabelecer e avaliar a relação entre tratores, energia, mão de obra e capital (terra e outros elementos) na produção agropecuária do estado. Essa também é uma informação importante para a elaboração de políticas para a gestão privada, posto que possa contribuir para políticas de orientação de médio e longo prazo na evolução da firma agropecuária. De uma perspectiva macroeconômica, à medida em que se identifica a inter-relação média presente na agropecuária, esforços no sentido de induzir modificações necessárias por crises e/ou conjunturas específicas serão mais profícuos, além de políticas para subsídios e investimentos.

Portanto, o objetivo deste artigo é estimar, a partir das informações dos municípios gaúchos, uma função custo da agropecuária gaúcha e, especificamente, calcular as elasticidades entre os insumos, apontando, notadamente, relações de complementaridade ou substituição.

A estimação será feita sob todo o espaço agropecuário gaúcho, um espaço heterogêneo onde unidades produtivas convivem com objetivos capitalistas, e outras são orientadas para a cooperação. Em paralelo, regiões do estado onde predominam pequenas e médias propriedades são consideradas em conjunto com regiões em que há a predominância de propriedades de grande porte.

Além desta introdução, na segunda seção, discutir-se-á o referencial teórico relacionado à metodologia de estimação da função de produção. Na terceira seção, os dados e os resultados da estimação são apresentados e discutidos em perspectiva e, na última, são realizados comentários conclusivos. 
Tabela 1. Evolução dos indicadores da agropecuária do Rio Grande do Sul

\begin{tabular}{|c|c|c|c|c|c|c|}
\hline & 1970 & 1975 & 1980 & 1985 & 1995 & 2006 \\
\hline $\begin{array}{l}\text { Área Total } \\
\text { (milhões de ha) }\end{array}$ & 23,8 & 23,6 & 24,0 & 23,8 & 21,8 & 19,7 \\
\hline $\begin{array}{l}\text { Lavouras } \\
\text { (milhões de ha) }\end{array}$ & 5,0 & 5,9 & 6,7 & 6,6 & 5,6 & 7,2 \\
\hline $\begin{array}{l}\text { Pastagens } \\
\text { (milhões de ha) }\end{array}$ & 14,6 & 13,7 & 13,3 & 12,9 & 11,7 & 8,9 \\
\hline $\begin{array}{l}\text { Matas } \\
\text { (milhões de ha) }\end{array}$ & 1,9 & 1,9 & 2,0 & 2,2 & 2,5 & 2,7 \\
\hline $\begin{array}{l}\text { Pessoal Ocupado } \\
\text { (milhões) }\end{array}$ & 1,446 & 1,893 & 1,747 & 1,747 & 1,377 & 1,219 \\
\hline Tratores & 39.923 & 77.254 & 120.070 & 138.540 & 150.074 & 161.434 \\
\hline Bovinos (milhões) & 12,305 & 12,692 & 13,985 & 13,509 & 13,221 & 11,148 \\
\hline Bubalinos & 1.596 & 3.464 & 11.762 & 23.328 & 65.917 & 50.976 \\
\hline Caprinos & 76.761 & 67.344 & 79.471 & 87.180 & 61.351 & 125.128 \\
\hline Ovinos (em mil unidades) & 12.157 & 11.361 & 10.696 & 8.394 & 5.081 & 3.326 \\
\hline $\begin{array}{l}\text { Suínos } \\
\text { (em milhões) }\end{array}$ & 5,851 & 5,612 & 5,421 & 4,225 & 3,933 & 5,827 \\
\hline $\begin{array}{l}\text { Aves } \\
\text { (em milhões) }\end{array}$ & 29,164 & 33,066 & 57,368 & 60,565 & 100,074 & 156,021 \\
\hline $\begin{array}{l}\text { Produção Leite Vaca } \\
\text { (em bilhões de litros) }\end{array}$ & 0,778 & 0,943 & 1,325 & 1,280 & 1,885 & 2,746 \\
\hline $\begin{array}{l}\text { Produção leite cabra } \\
\text { (em milhões de litros) }\end{array}$ & - & 156 & 381 & 313 & 345 & 591 \\
\hline $\begin{array}{l}\text { Produção de lã } \\
\text { (toneladas) }\end{array}$ & 33.216 & 31.202 & 29.666 & 23.336 & 12.957 & 10.074 \\
\hline Ovos (milhões de dúzias) & 56,395 & 74,497 & 127,123 & 132,344 & 225,628 & 388,765 \\
\hline
\end{tabular}

Fonte: Dados da Tabela 1.3.17 em IBGE (2007) 
Tabela 2. Índice de evolução técnica da agropecuária do Rio Grande do Sul

\begin{tabular}{|c|c|c|c|c|c|c|}
\hline & 1970 & 1975 & 1980 & 1985 & 1995 & 2006 \\
\hline [Pessoal Ocupado / Área de lavouras] & 0,29 & 0,32 & 0,26 & 0,26 & 0,24 & 0,17 \\
\hline [Tratores / Área de lavouras] (x 1000) & 8,02 & 13,03 & 17,97 & 21,01 & 26,63 & 22,30 \\
\hline [Bovinos / Área de pastagens] & 0,84 & 0,92 & 1,05 & 1,04 & 1,13 & 1,24 \\
\hline Unidade Animal² & 1,19 & 1,09 & 0,95 & 0,96 & 0,88 & 0,80 \\
\hline [Pessoal Ocupado / Tratores] & 36,24 & 24,52 & 14,55 & 12,62 & 9,18 & 7,55 \\
\hline
\end{tabular}

Fonte: Dados elaborados a partir de números da Tabela 1.3.17 em IBGE (2007)

\section{Metodologia e base de dados}

"Algumas análises que estimam funções de produção podem ser encontradas na literatura. Dos trabalhos pioneiros, compilados por Barbosa (1985), destacam-se: Rocca(1970), Goodman et al. (1971), Macedo (1974), Scandizzo e Albuquerque (1977) e Luque (1977). Recentemente, Parré e Ferreira Filho (1998), Gasques et al. (2004), Freitas e Bacha (2004) e Almeida (2005).”

Outros estudos que exploram a dualidade entre funções de produção e função de custo, e que contemplam a estimação de funções custo para diferentes objetivos, podem ser encontrados em LAU e YOTOPOULOS (1971, 1972), Antle (1984), Albuquerque (1987) e Garcia e Ferreira Filho (2004), para a produção de frangos de corte, Conte e Ferreira Filho (2007), para a produção de soja no estado do Mato Grosso, Gomes e Ferreira Filho (2007), que estimam ganhos de economia de escala para a produção de leite nos estados do Tocantins, Rondônia e Rio de Janeiro e Ishii et al. (2007), que estimam uma função lucro para a produção de soja no Mato Grosso. Alguns, como Antle (1984), utilizam-se de séries em painel, ao passo que a maior parte dos estudos brasileiros dedica-se a estudos em cross-section. Barbosa (1985) também ilustra algumas das aplicações para o Brasil. Por vezes, o objetivo não é apenas extrair as relações de elasticidades entre os insumos, mas também obter as possibilidades de ganho de escala em propriedades. Como na amostra desse artigo trabalhamos com municípios, a possibilidade de se calcular escalas produtivas ótimas foi descartada, restando, no entanto a oportunidade de cálculo das elasticidades dos insumos.

Em uma variante, Sidhu e Baanante (1981) também calculam a elasticidade de demanda por insumos e a elasticidade de oferta para uma amostra de produtores do Punjab indiano por meio do uso da função lucro. Mullibeaux (1978) também 
pela ótica dessa função constrói uma análise para o setor bancário. Ainda, como lembram Mcfadden (1978), Chambers (1988), Silberberg (1990) e Varian (1992), há equivalência entre a estimação da função lucro e da função custo, tal como existe entre a função de produção e a função custo, também.

Ainda, Soares et al (2007) estimam uma função de produção para o setor de celulose brasileiro, considerando a terra, o trabalho e o capital como fatores de produção. Porém, ao invés de uma forma flexível, os autores utilizam-se de uma função Cobb-Douglas. Almeida (2005) dedica-se à estimação da função agropecuária brasileira, incluindo aspectos relativos à logística e aos efeitos de transbordamento regional.

Para estimar a função de produção, no entanto, será utilizada a função translog - transcendental logarítmica. Sua aplicabilidade reside em ser flexível, ou seja, sem determinar uma especificação a priori, ao mesmo tempo em que permite aproximar uma função arbitrária contínua e duplamente diferenciável (Chambers, 1988). A equação (1) já apresenta o formato que será utilizado neste artigo, contendo os insumos objetos de estudo.

Seu formato é dado pelos logaritmos da expansão da série de Taylor:

$$
\begin{aligned}
\ln y=\beta_{0}+ & \beta_{A} \ln A+\beta_{C} \ln C+\beta_{L} \ln L+\beta_{T} \ln T+1 / 2 \beta_{A A}(\ln A)^{2} \\
& +1 / 2 \beta_{C C}(\ln C)^{2}+1 / 2 \beta_{L L}(\ln L)^{2}+1 / 2 \beta_{T T}(\ln T)^{2} \\
& +1 / 2 \varphi_{A C}(\ln A)(\ln C)+1 / 2 \varphi_{A L}(\ln A)(\ln L) \\
& +1 / 2 \varphi_{A T}(\ln A)(\ln T)+1 / 2 \varphi_{C L}(\ln C)(\ln L) \\
& +1 / 2 \varphi_{C T}(\ln C)(\ln T)+1 / 2 \varphi_{L T}(\ln L)(\ln T)+\varepsilon_{i}
\end{aligned}
$$

Em que:

Y - produção agrícola (em mil reais), no município, ano de 2006;

L - total de mão de obra dedicada à agropecuária no município, ano de 2006 ;

$\mathrm{T}$ - total de tratores no município, ano de 2006;

$\mathrm{C}$ - total de insumos para preparação do solo e conservação da atividade agropecuária, ano de 2006;

A - total das áreas temporárias e permanentes dedicada à agropecuária no município (em hectares), ano de 2006;

Ln - logaritmo neperiano;

$\varepsilon_{\mathrm{i}}$ - erro de estimativa. 
As derivadas primeiras e segundas em $\ln ()=$.0 , seriam:

$$
\begin{aligned}
& \frac{\partial \ln y *}{\partial \ln C}=(y / A)\left[\beta_{A}+\beta_{A A}(\ln A)+1 / 2 \varphi_{A C}(\ln C)+1 / 2 \varphi_{A L}(\ln L)+1 / 2 \varphi_{A T}(\ln T)\right] \\
& \frac{\partial \ln y *}{\partial \ln C}=(y / C)\left[\beta_{C}+\beta_{C C}(\ln C)+1 / 2 \varphi_{A C}(\ln A)+1 / 2 \varphi_{C L}(\ln L)+1 / 2 \varphi_{C T}(\ln T)\right] \\
& \frac{\partial \ln y *}{\partial \ln L}=(y / L)\left[\beta_{L}+\beta_{L L}(\ln L)+1 / 2 \varphi_{A L}(\ln A)+1 / 2 \varphi_{C L}(\ln C)+1 / 2 \varphi_{L T}(\ln T)\right] \\
& \frac{\partial \ln y *}{\partial \ln T}=(y / T)\left[\beta_{T}+\beta_{T T}(\ln T)+1 / 2 \varphi_{A T}(\ln A)+1 / 2 \varphi_{C T}(\ln C)+1 / 2 \varphi_{L T}(\ln L)\right]
\end{aligned}
$$

Para se obter as elasticidades há, que se considerar a taxa marginal de substituição técnica (TMST) entre os insumos, e, contrariamente à Barbosa (1985), não iremos considerar $\beta_{I I}=\beta_{I L}=\beta_{L L}=\beta$, ou, em outras palavras, que a função seja necessariamente homogênea. Tal resultado, entretanto, pode surgir a partir das estimativas, de modo que os resultados para as TMSTs são:

$$
\begin{gathered}
\operatorname{TMST}_{A C}=(C / A)\left[\frac{\beta_{A}+\beta_{A A}(\ln A)+1 / 2 \varphi_{A C}(\ln C)+1 / 2 \varphi_{A L}(\ln L)+1 / 2 \varphi_{A T}(\ln T)}{\beta_{C}+\beta_{C C}(\ln C)+1 / 2 \varphi_{A C}(\ln A)+1 / 2 \varphi_{C L}(\ln L)+1 / 2 \varphi_{C T}(\ln T)}\right] \\
\operatorname{TMST}_{A L}=(L / A)\left[\frac{\beta_{A}+\beta_{A A}(\ln A)+1 / 2 \varphi_{A C}(\ln C)+1 / 2 \varphi_{A L}(\ln L)+1 / 2 \varphi_{A T}(\ln T)}{\beta_{L}+\beta_{L L}(\ln L)+1 / 2 \varphi_{A L}(\ln A)+1 / 2 \varphi_{C L}(\ln C)+1 / 2 \varphi_{L T}(\ln T)}\right] \\
\operatorname{TMST}_{A T}=(T / A)\left[\frac{\beta_{A}+\beta_{A A}(\ln A)+1 / 2 \varphi_{A C}(\ln C)+1 / 2 \varphi_{A L}(\ln L)+1 / 2 \varphi_{A T}(\ln T)}{\beta_{T}+\beta_{T T}(\ln T)+1 / 2 \varphi_{A T}(\ln A)+1 / 2 \varphi_{C T}(\ln C)+1 / 2 \varphi_{L T}(\ln L)}\right] \\
\operatorname{TMST}_{C L}=(L / C)\left[\frac{\beta_{C}+\beta_{C C}(\ln C)+1 / 2 \varphi_{A C}(\ln A)+1 / 2 \varphi_{C L}(\ln L)+1 / 2 \varphi_{C T}(\ln T)}{\beta_{L}+\beta_{L L}(\ln L)+1 / 2 \beta_{A L}(\ln A)+1 / 2 \varphi_{C L}(\ln C)+1 / 2 \varphi_{L T}(\ln T)}\right] \\
T_{M S T_{C T}}=(T / C)\left[\frac{\beta_{C}+\beta_{C C}(\ln C)+1 / 2 \varphi_{A C}(\ln A)+1 / 2 \varphi_{C L}(\ln L)+1 / 2 \varphi_{C T}(\ln T)}{\beta_{T}+\beta_{T T}(\ln T)+1 / 2 \varphi_{A T}(\ln A)+1 / 2 \varphi_{C T}(\ln C)+1 / 2 \varphi_{L T}(\ln L)}\right]
\end{gathered}
$$




$$
T M S T_{L T}=(T / L)\left[\frac{\beta_{L}+\beta_{L L}(\ln L)+1 / 2 \varphi_{A L}(\ln A)+1 / 2 \varphi_{C L}(\ln C)+1 / 2 \varphi_{L T}(\ln T)}{\beta_{T}+\beta_{T T}(\ln T)+1 / 2 \varphi_{A T}(\ln A)+1 / 2 \varphi_{C T}(\ln C)+1 / 2 \varphi_{L T}(\ln L)}\right]
$$

Com base nas taxas marginais de substituição técnica, é possível recordar a fórmula da elasticidade entre os fatores, genericamente expressa, conforme Silberberg (1990):

$$
\sigma=-\left[\frac{d\left(x_{2} / x_{1}\right)}{d\left(f_{1} / f_{2}\right)}\right]\left[\frac{f_{1} / f_{2}}{x_{2} / x_{1}}\right]
$$

Então,

$$
\sigma_{i i}=-\frac{f_{i} f_{j}\left(x_{i} f_{i}+x_{j} f_{j}\right)}{x_{i} x_{j}\left(f_{i i} f_{j}^{2}-2 f_{i j} f_{i} f_{j}+f_{j j} f_{i}^{2}\right.}
$$

Ressalte-se que a homogeneidade linear da função de produção translog requer que a somatória dos coeficientes $\beta_{\mathrm{i}}$ sejam iguais a 1 e que a soma dos coeficientes $\psi_{\mathrm{xz}}$ em X sejam iguais a zero, assim como a soma dos coeficientes $\psi_{\mathrm{xz}}$ em X e em Z. Neste artigo, não se assumirá a homogeneidade linear como um resultado dado, sendo, entretanto, possível testá-la a partir dos resultados da estimação.

A amostra será composta por cada um dos municípios gaúchos em que registrou-se algum produto agropecuário no ano de 2006. Assim, o critério de produto final (Y) utilizado nessa estimação é o valor da produção agrícola no ano de 2006 (em milhares de reais).

Os dados obtidos do IBGE (2007) permitem dividir o problema em alguns insumos típicos. Pensando-se a produção da propriedade agrícola, localizamos alguns insumos candidatos. Uma vez que o Censo traz informações múltiplas, preferiu-se agregar vários desses insumos em grandes representações, como detalhamos a seguir.

Um primeiro insumo necessário ao processo produtivo agrícola é a terra. As informações do censo detalham a divisão municipal em área de lavoura temporária e área de lavoura permanente. Neste estudo, preferiu-se agregar as duas tipologias em um único insumo correspondente à área de produção.

Um segundo insumo presente é a mão de obra, que é utilizada em todos os estágios de produção. Nesse caso, preferiu-se somar o número de trabalhadores homens e mulheres, temporários e permanentes, com mais de 14 anos de idade. 
Nessa estatística estão incluídas as pessoas que fazem parte da família do proprietário da terra, e, por vezes, não recebem remuneração monetária e/ou direta. De todo modo, são pessoas que colaboram na obtenção do produto final e, consequentemente, estão consideradas na função de produção.

Um terceiro insumo considerado na produção foi o número de tratores, no qual calculou-se o número de tratores com potência superior a $100 \mathrm{hp}$ e potência inferior $100 \mathrm{hp}$, estabelecendo uma classe única de insumos. Destaque-se, porém, que em todos os municípios que compõe a amostra há a predominância de tratores com potência inferior a $100 \mathrm{hp}$.

Um último insumo refere-se aos instrumentos utilizados nas três etapas principais de produção: semeadura, desenvolvimento e colheita.. As informações disponibilizadas pelo Censo são disponibilizadas em número de unidades nos municípios. Assim, optou-se por somar as quantidades de arados, semeadeiras e adubadeiras (semeadura) aos de enxadas rotativas, roçadeiras e pulverizadeiras (desenvolvimento).

Desse modo, teremos quatro insumos: terra, tratores, instrumentos e trabalho e, como produto, o valor da produção (em mil reais). Como exigido pela função trans-log, os dados foram transformados em logaritmo, além da inclusão dos termos quadráticos e termos cruzados para cada unidade da amostra em 2006.

\section{Estimação e resultados}

Nesta seção, pretende-se expor a estimação e os problemas que foram contornados, além dos resultados obtidos. Na seção metodológica ficou claro a importância de posse dos valores e significâncias obtidos nos parâmetros para recuperar o formato da função de produção, bem como da possibilidade de se examinar as elasticidades de substituição ou complementaridade entre os fatores de produção. 
Tabela 3. Resultados da estimação do modelo da equação (6) - função trans-log para a agropecuária gaúcha

\begin{tabular}{|c|c|c|c|c|c|c|}
\hline & \multirow{2}{*}{ Coeficiente } & \multirow{2}{*}{ Desvio padrão } & \multirow{2}{*}{ T - Robusto } & \multirow{2}{*}{ Prob. } & \multicolumn{2}{|c|}{ Intervalo de confiança à 95\% } \\
\hline & & & & & Mín. & Máx. \\
\hline$\beta_{0}$ & $-0,7604$ & 0,3154 & $-2,41$ & 0,016 & $-1,380$ & $-0,140$ \\
\hline$\beta_{\mathrm{A}}$ & 0,4992 & 0,1789 & 2,79 & 0,005 & 0,1478 & 0,8507 \\
\hline$\beta_{\mathrm{L}}$ & 2,7345 & 0,3297 & 8,30 & 0,000 & 2,0868 & 3,3823 \\
\hline$\beta_{\mathrm{T}}$ & 0,5932 & 0,3748 & 1,58 & 0,114 & $-0,143$ & 1,329 \\
\hline$\beta_{c}$ & $-1,6075$ & 0,4610 & $-3,49$ & 0,001 & $-2,513$ & $-0,701$ \\
\hline$\beta_{\mathrm{AA}}$ & 0,1623 & 0,0468 & 3,47 & 0,001 & 0,070 & 0,254 \\
\hline$\beta_{\mathrm{LL}}$ & $-0,0886$ & 0,2144 & $-0,41$ & 0,679 & $-0,510$ & 0,332 \\
\hline$\beta_{\mathrm{TT}}$ & 0,2563 & 0,1181 & 2,17 & 0,030 & 0,0242 & 0,4883 \\
\hline$\beta_{\mathrm{cc}}$ & 0,1092 & 0,2513 & 0,43 & 0,664 & $-0,384$ & 0,6030 \\
\hline$\Psi_{\mathrm{AL}}$ & $-0,7305$ & 0,1656 & $-4,41$ & 0,000 & $-1,055$ & $-0,405$ \\
\hline$\Psi_{\text {AT }}$ & 0,7236 & 0,1211 & 5,98 & 0,000 & 0,485 & 0,961 \\
\hline$\Psi_{\mathrm{AC}}$ & $-3,8149$ & 0,1901 & $-2,01$ & 0,045 & $-0,755$ & $-0,007$ \\
\hline$\Psi_{\mathrm{LT}}$ & $-0,8533$ & 0,2635 & $-3,24$ & 0,001 & $-1,371$ & $-3,356$ \\
\hline$\Psi_{\mathrm{LC}}$ & 1,0759 & 0,4170 & 2,58 & 0,010 & 0,256 & 1,895 \\
\hline$\Psi_{\mathrm{TC}}$ & $-0,4471$ & 0,2653 & $-1,69$ & 0,093 & $-0,968$ & 0,074 \\
\hline
\end{tabular}

Fonte: Resultados do Modelo

A estimação da Tabela 3 deu-se por meio de um estimador de máxima verossimilhança, contornando problemas de heterocedasticidade que pudessem comprometer a confiabilidade dos intervalos.

Conforme Chambers (1988), os parâmetros obtidos na estimação da função trans-log não são ainda suficientes, embora necessários, para o estabelecimento das relações entre os insumos. Essa relação, em particular a sua significância, contudo, só pode ser obtida a partir dessa primeira estimativa, observando as equações apresentadas entre (6) e (13).

A Tabela 4 apresenta as estatísticas descritivas relacionadas às variáveis utilizadas. Como se pode perceber, consideraram-se os 496 municípios do estado, em sua totalidade, o que é uma limitação, uma vez que as microrregiões têm perfis distintos de produção, especialmente na metade do Sul, dedicada à pecuária, e na metade Norte, dedicada à agricultura de produção de grãos, por exemplo. 
Tabela 4. Valores médios em log dos insumos

\begin{tabular}{l|r|r|r|r}
\hline \multirow{2}{*}{ Variável } & \multirow{2}{*}{$N^{\circ}$ de observações } & Média aritmética & \multicolumn{2}{c}{ Intervalo de confiança à 95\% } \\
\cline { 4 - 5 } & & & Mín. & \multicolumn{1}{c}{ Máx. } \\
\hline A & 496 & 3,946648 & 3,890608 & 4,002687 \\
C & 496 & 2,945545 & 2,908911 & 2,982179 \\
L & 496 & 3,229364 & 3,195193 & 3,263534 \\
T & 496 & 2,32573 & 2,287937 & 2,363524 \\
\hline
\end{tabular}

Fonte: Resultados do modelo

Finalmente, na Tabela 5, surgem os resultados relativos à relação entre os insumos com base nas equações (6) a (11). Sobretudo, relações elásticas de substituição surgem entre outros insumos e tratores, e desse último elemento com relação à área. Denota a Tabela 5, sobretudo, as oportunidades de incremento da produtividade na agricultura gaúcha cujo espaço geográfico também se apresenta limitado. Outra relação de substituição surge na relação entre outros insumos e trabalho, porém, dessa vez, de maneira inelástica. As relações de complementariedade são todas inelásticas e se dão entre área e outros insumos, área e trabalho; e trabalho e tratores.

Tabela 5. Resultados da estimação do modelo da equação (1) - elasticidade de substituição pelas equações (6) - (11)

\begin{tabular}{l|r}
\multicolumn{1}{c|}{ Relação } & Elasticidade de substituição \\
\hline Área - Insumos produção & $-0,61477$ \\
Área - Trabalho & $-0,26653$ \\
Área - Tratores & 1,113544 \\
Insumos produção - Trabalho & 0,232127 \\
Insumos produção - Tratores & 20,83218 \\
Trabalho - Tratores & $-0,56822$ \\
\hline
\end{tabular}

Fonte: Resultados do modelo

A Figura 1 tenta sintetizar as relações encontradas na função de produção da agropecuária no Rio Grande do Sul obtidas na Tabela 5. Enquanto nessa tabela há valores numéricos, consequência das estimações econométricas, a figura tenta expressar as relações de um ponto de vista qualitativo e inter-relacionar os insumos pesquisados. 


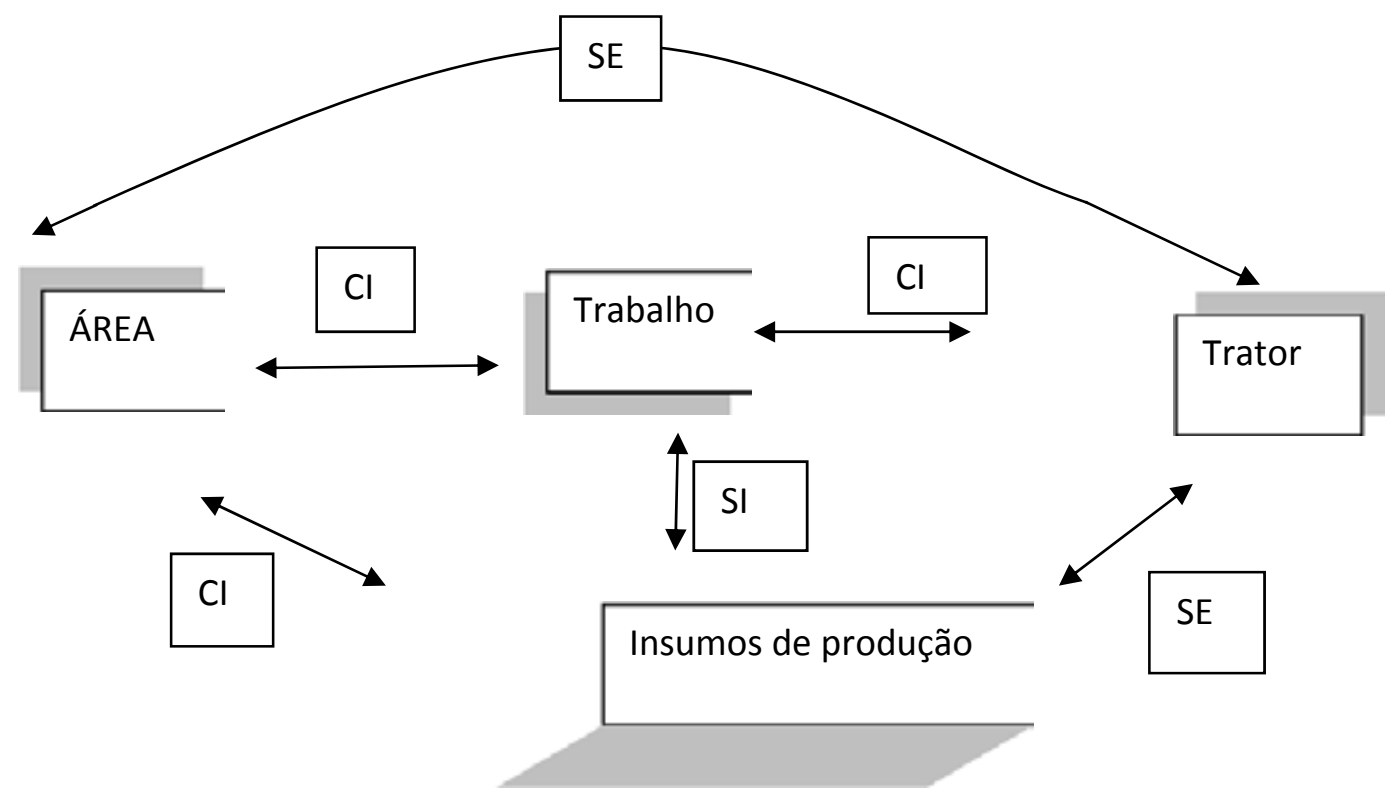

Fonte: Elaboração Própria; Legenda: $\mathrm{Cl}$ - complementariedade inelástica, SE - substituição elástica, SI - substituição inelástica.

Figura 1. Resumo das relações obtidas entre os insumos da agropecuária do Rio Grande do Sul 2006

\section{Comentários finais}

Percebe-se, pela estimação realizada neste artigo, algumas relações entre os insumos utilizados na agropecuária nos municípios gaúchos. Com base nos dados do Censo Agropecuário de 2006, é possível notar algumas características da produção local.

Assim, verifica-se que, no estado do Rio Grande do Sul, a agropecuária tem potenciais interessantes, explorando a complementaridade e a substituição entre os insumos. A característica de complementariedade entre trabalho e tratores indica um resultado importante, já que se percebe uma interação e a possibilidades de ganhos produtivos conjuntos, indicando a oportunidade de aproveitamento de um trabalhador mais especializado. Ademais, essa condição no nível dos municípios, em 2006, complementa a percepção da trajetória dos dados temporais entre 1995 e 2006. A relação de substituição entre área e trator também assinala o processo de intensificação da produtividade, sobretudo em um estado onde as oportunidades de fronteira agrícola estão essencialmente encerradas. 
Os resultados podem ser úteis, também, para o cálculo da escala produtiva ótima. Entretanto, acreditamos que para tal, seria necessário dispor dos dados no nível das propriedades, ao invés dos municípios. Também não podemos afirmar que os resultados aqui obtidos sejam passíveis de extrapolação para outros estados, necessitando-se uma análise particular caso a caso, em especial para os estados com geografia e dinâmica econômica distintas das do Rio Grande do Sul. Todavia, foi possível determinar como se dá a relação entre os insumos mais importantes utilizados na agricultura gaúcha, podendo essa informação pode ser útil para a definição de políticas agrícolas para o Estado.

No entanto, cabe destacar que as estimativas foram construídas para uma amostra estadual, sem considerar idiossincrasias regionais ou características produtivas locais, contemplando a heterogeneidade estadual. Estudos futuros, em que pese a redução da amostra, podem contemplar macrorregiões e/ou microrregiões particulares, contribuindo para aumentar a compreensão sobre as estruturas da agropecuária gaúcha. 


\title{
The Production Function in agriculture sector of Rio Grande do Sul in 2006
}

\begin{abstract}
This paper purpose a estimation of production function in agricultural sector at Rio Grande do Sul municipalities, 2006, from Brazilian agricultural census. From production function is feasible find complementary and substation elasticity between production factors. The relations show inelastic complementary relation among land and labor, labor and machines, land and other inputs. Substitution inelastic elasticity among land and other inputs is finding also. Finally, substitution elastic elasticity is find amid land and machinery, machinery and other inputs.
\end{abstract}

Keywords: Agriculture, Production Function, Rio Grande do Sul, Substitution Elasticity

\section{La función de producción en la agricultura Gaucha en 2006}

\section{Resumen}

En este trabajo se estima una función de producción para la agricultura del Rio Grande do Sul del Censo 2006 en los municipios. En particular, a partir de la función de producción estimada se obtienen las relaciones de complementariedad y sustitución entre factores primarios de producción. Sorprendentemente, se obtienen las relaciones de complementariedad inelásticas entre el área y el trabajo, el trabajo y los tractores, el área y otros insumos. Una relación de substitución inelástica se encuentra con el trabajo y otros insumos. Por último, tenga en cuenta la tasa de sustitución elástica para el área y tractores y, tanbién para tractores y otros insumos.

Palabras clave: Agricultura. Función de producción. Rio Grande do Sul. Elasticidades de sustitución. 


\section{Notas}

1 Neste valor foram somadas as atividades agrícolas, silvicultura e exploração florestal, além de pecuária.

2 Esta é uma medida inversa à linha anterior. Portanto, corresponde à área necessária para uma cabeça.

\section{Referências}

ALBUQUERQUE, M. C. C. Uma análise translog sobre mudança tecnológica e efeitos de escala: um caso de modernização eficiente. Pesquisa e Planejamento Econômico, Rio de Janeiro, v. 1, n. 17, p. 191-220, 1987.

ALMEIDA, E. S. Função de produção agropecuária espacial. Ribeirão Preto, Anais do XLIII Congresso da Sociedade Brasileira de Economia, Administração e Sociologia Rural, 2005.

ANTLE, J. M. The structure of U.S. agricultural technology, 1910-78. American Journal of Agricultural Economics, Malden, v. 66, n. 4, p. 414-421, Nov., 1984.

BARBOSA, F. H. Microeconomia: teoria, modelos econométricos e aplicações à economia brasileira. Rio de Janeiro: IPEA/INPES, 1985, 534 p.

CHAMBERS, R. G. Applied production analysis: a dual approach. Cambridge: Cambridge University Press, 1988, 331 p.

CHRISTENSEN, L. R.; JORGENSON; D. W.; LAU L. J. Conjugate duality and the transcedental logarithmic function. Econometrica, v. 39, n. 3, p. 255-256, jul. 197.

Transcedental logarithmic production frontiers. The Review of Economics and Statistics, v. 55, n. 1, p. 28-45, Feb. 1973

CONTE, L.; FERREIRA FILHO, J. B. S. Substituição de fatores produtivos na produção de soja no Brasil. Revista de Economia e Sociologia Rural, Piracicaba, v. 45, p. 475, 2007

FREITAS, C. A.; BACHA, C. J. C. Contribuição do capital humano para o crescimento da agropecuária brasileira - período de 1970 a 1996. Revista Brasileira de Economia, Rio de Janeiro, v. 58, n. 4, p. 533-557, 2004.

GARCIA, L. A. F.; FERREIRA FILHO, J. B. S. Economias de Escala na Produção de Frangos de Corte no Brasil. Revista de Economia e Sociologia Rural, Brasília, v. 43, n.3, p. 465-484, 2005.

GASQUES, J. C.; et al. Desempenho e crescimento do agronegócio no Brasil. Brasília: IPEA, 2004. (Texto para Discussão no 1009). Disponível em: <http://www.ipea.gov.br/pub/td/2004/ td_1009.pdf $>$.

GOMES, A. L. ; FERREIRA FILHO, J. B. S. . Economias de escala na produção de leite: uma análise dos estados de Rondônia, Tocantins e Rio de Janeiro. Revista de Economia e Sociologia Rural, Piracicaba, v. 45, p. 591-620, 2007.

GOODMAN, D. E.; SENA, J. F. F.; ALBUQUERQUE, R. C. Os incentivos financeiros à industrialização do Nordeste e a escolha de tecnologias. Pesquisa e Planejamento, Rio de Janeiro, v. 1, n. 2, p. 329-366, 1971.

GREENE, W. H. Econometric Analysis. New Jersey: Prentice-Hall, 2000. 1004p.

Teoria e Evidência Econômica - Ano 19, n. 41, p. 102-117, jul./dez. 2013 
INSTITUTO BRASILEIRO DE GEOGRAFIA E ESTATÍSTICA - IBGE. Censo Agropecuário 2006: resultados preliminares. IBGE: Rio de Janeiro, 2007.

INSTITUTO BRASILEIRO DE GEOGRAFIA E ESTATÍSTICA - IBGE. Matriz insumo-produto 2005. Rio de Janeiro: IBGE, 2008.

INSTITUTO BRASILEIRO DE GEOGRAFIA E ESTATÍSTICA - IBGE. Contas Nacionais e Regionais 2009. Rio de Janeiro: IBGE, 2011.

ISHII, K. S.; SOUZA, M. J. P.; FERREIRA FILHO, J. B. S. A oferta e a estrutura de demanda de fatores de produção da sojicultura brasileira: o modelo da função lucro translog. In: Anais do Congresso Brasileiro de Economia e Sociologia Rural, 2007, Londrina, 2007

LAU, L. J.; YOTOPOULOS, P. A. A test for relative Efficiency and an application to Indian agriculture. American Economic Review, v. 61, p. 94-109, March, 1971.

LUQUE, C. A. Elasticidade de escala e taxa efetiva de incentives à exportação. Pesquisa e Planejamento Econômico, Rio de Janeiro, v. 7, n. 2, p. 405-422, 1977.

McFADDEN, D. Cost, revenue and profit function in production functions: a dual approach to theory and applications. In: FUSS, M. e MCFADDEN, D. (Orgs.), Nova York, North-Holland, 1978.

MULLIBEAUX D. J. Economies of scale and organizational efficiency in banking: A profit function approach. The Journal of Finance, v. 33, n. 1, p. 259-280, March, 1978.

PARRÉ, J. L.; FERREIRA FILHO, J. B. S. Estudo da tecnologia utilizada na produção de soja no Estado de São Paulo. Revista Teoria e Evidência Econômica, Passo Fundo, v. 6, n. 11, p. 7-18, nov. 1998.

ROCCA, C. A. Productivity in Brazilian manufacturing. In: Bergsman, J. (Org.). Brazil: industrialization and trade policies. Cambridge: Oxford University Press, p. 222-241, 1970.

SCANDIZZO, P. L.; BARBOSA, T. Substituição e produtividade de fatores na agricultura nordestina. Pesquisa e Planejamento Econômico, Rio de Janeiro, v. 7, n. 2 , p. 367-404, 1977.

SIDHU, S. S.; BAANANTE, C. Estimating farm-level input demand and wheat supply in the Indian punjab using a translog profit function. American Journal of Agricultural Economics, Malden, v. 63, n. 2, p. 237-246, 1981.

SILBERBERG, E. The structure of economics: a mathematical analysis. 2. ed., Nova York, McGraw-Hill, 1990. 686 p.

SOARES, N. S.; SILVA, Márcio L.; lima, João E. A função de produção da indústria brasileira de celulose em 2004. Viçosa, Revista Árvore, v. 31, n. 3, p. 495-502, 2007.

VARIAN, H. R. Microeconomic Analysis, 3. ed.. New York: W.W. Norton, 1992, 556 p. 